\title{
Cognitive-behaviour therapy and schizophrenia
}

\author{
Til Wykes
}

Institute of Psychiatry, King's College London, London, UK

Correspondence to Til Wykes, t.wykes@kcl.ac.uk

\begin{abstract}
People who experience debilitating psychotic symptoms that affect their everyday life are often, but not always, given a diagnosis of schizophrenia. Although the first line of treatment is medication, many people experience a suboptimal response and after the acute symptoms resolve they can continue to experience both hallucinations and delusions. These are generally termed residual symptoms and are the phenomena that cognitivebehavioural therapy for psychosis (CBTp) was originally devised to target. The success of CBTp in randomised controlled trials from the early 90 s and evidence of cost-effectiveness has meant that many healthcare services across the world include CBTp in their treatment armamentaria. For instance, in the UK the National Institute for Health and Care Excellence guidance says that all individuals who have a diagnosis of schizophrenia should be given the option of a course of CBTp. Recently, however, the treatment effects have been re-examined, the targets widened and the premise that CBTp should be solely an adjunct to medication has been questioned. This article will describe and probe some of these changes and reflect on the development of psychological treatments for psychosis.
\end{abstract}

\section{WHAT IS COGNITIVE-BEHAVIOURAL THERAPY FOR PSYCHOSIS?}

As cognitive-behavioural therapy for psychosis (CBTp) expands to address other targets, it is important to consider its origins and vital ingredients.

CBTp is a verbal therapy to ease distress by reducing positive symptoms. It does this by mobilising the client's capacity to reflect on and to question delusional or self-evaluative beliefs through a 'collaborative empirical' enterprise. The therapist joins forces with the client to question beliefs that limit the achievement of personal life goals. The journey through therapy (usually 20 or so sessions over 6-9 months) allows for the collaborative development of an understanding of distressing psychotic experiences. The clients are then guided to re-evaluate their appraisals of experiences and identify new ways of responding to them. Towards the end of therapy further collaborative work on maintaining factors is carried out to support the individual to prevent relapse. Usually this involves issues such as reasoning style, selfconcept, social isolation, appraisals of psychosis and emotional processes. Models are provided for therapy development ${ }^{1}$ and all therapists are expected to cultivate a shared formulation of the relationship between the experiences, the thoughts and the problematic behaviour. In this paragraph I have tried to describe what in many books takes more than 300 pages and usually months of therapy training to achieve delivery competence (although the level of skills required is debated). ${ }^{2-}$

${ }^{5}$ The introduction of this therapy was a turning point in mental healthcare delivery as it advocated discussing hallucinations and delusions which, until the emergence of this therapy, was thought to constitute a poor approach to care.

\section{THERAPY EFFECTIVENESS}

The gauge of therapy effectiveness is usually a meta-analysis. These studies follow rules now described in the Preferred Reporting Items for Systematic Reviews and Meta-Analyses (PRISMA) guidelines which require authors to specify their inclusion criteria (therapy description, participants and target outcomes), data extraction, data quality checks, etc. Although following these rules allows for robust replication, it is still important that the research team has access to therapeutic expertise in order to detect true trials of the therapy under investigation. All meta-analyses also depend on the level of development of the therapy so that early in development there will be more pilot or exploratory trials that may be open to bias. However, after multiple replications by different groups, we may be more certain that the results are true effects.
One meta-analysis carried out a strict investigation of CBTp with 25 trials of the effects on one outcome-positive symptoms - which was the target designated for the original development of CBTp. ${ }^{4}$ This meta-analysis also reported on the overall effects after considering those studies with a high likelihood of bias using a scale formatted specifically for psychological treatment trials. As might be expected, the effect size from trials with a low risk of bias was smaller than that for studies with a high risk, but it was still significant. This same meta-analysis also demonstrated that individual assessors being blind to the treatment allocation was the most significant predictor of the bias. This is not a new finding and has been reported for treatments in many other disorders and for other treatments such as medication. So this might be thought of as a slam dunk and we do not need to do any more, but recently a series of meta-analyses were produced investigating current data and testing both the expansion of CBTp to targets other than positive symptoms as well as comparing CBTp with other treatments.

CBTp has expanded to include targets such as negative symptoms, ${ }^{6}$ social outcomes ${ }^{7}$ and compliance with command hallucinations, ${ }^{8}$ among many others. The formulation-based approach which is a highlight of this therapy has led to subtle adaptations which allow a clear movement towards these target-based goals. This, of course, leads to outcomes that are tailored and are usually not measured as traditional positive symptoms and the trials are powered for these new outcomes. It is therefore vital to ensure that in any meta-analysis the right outcomes are compared. The 'washing machine' approach of meta-analyses throwing together studies targeting differing outcomes but measuring the same one can produce some interesting data on effectiveness. $^{9}$ If it is positive, then this suggests that all CBTs can affect a variety of outcomes (the target and others), but if effectiveness in a 'washing machine' meta-analysis shows a negative result, how must we interpret this result? Obviously the therapy approaches are not equivalent for all outcomes and possibly a tailored approach would be more effective. Luckily the recent published meta-analyses can throw some light on these effects. All are rigorous and are carried out according to the PRISMA guidance and all include sensitivity to the likelihood of bias resulting from poorer methods.

\section{THE STORY SO FAR. .}

This year Jauhar et al ${ }^{10}$ included a wide variety of studies - 34 in all. This meta-analysis emphasised the overall value of CBTp approaches to both positive and negative symptoms derived from assessor interviews. However, the inclusion criteria did exclude some targeted therapies, in particular those that targeted hallucinations. In their analyses they 
discovered a benefit of CBTp across all studies but when they considered only those with blinded assessment the $\mathrm{Cl}$ crossed zero. Burns et $\mathrm{l}^{11}$ also collated studies with all targets but specified a further inclusion criterion-positive symptoms that were medication resistant. They found a benefit (effect size $=0.43 ; 95 \% \mathrm{Cl} 0.20$ to 0.67 ) for studies with blinded group allocation. So what are we to make of the different results? Clearly with a specific group-those with medication resistant symptoms - we can reasonably assume that there is a modest effect and this is understandable as this was the original remit for the development of CBTp. The study by van der Gaag et al ${ }^{12}$ supports this conclusion as their meta-analysis disaggregated delusions and hallucinations as outcomes and found a benefit of CBTp to both targets even after taking into account blinded assessment. So therapy benefits may be dependent on the specific target, how these are measured and the types of individuals included in the studies.

\section{IS CBTP DIFFERENT FROM ANY OTHER PSYCHOLOGICAL TREATMENT?}

This is an important question as it affects treatment guidance in general and the necessity for training for more complex therapies. It is answered in a paper comparing psychological treatments such as social skills, cognitive remediation, CBTp, befriending, psychoeducation and supportive counselling. ${ }^{13}$ In the set of studies with a low risk of bias, CBTp was the most effective for positive symptoms (effect size $=0.16$ ) compared to all other treatments. So there are differences in efficacy of psychological treatments for psychosis which can guide treatment choice which should depend on what individual patients select as their main goal. Differences in effectiveness found in this meta-analysis are consistent with the specific factors in the interventions and their specific targets in particular.

\section{IS CBTP STANDING STILL?}

Two further meta-analyses published in the past year suggest even more ambitious targets. These meta-analyses investigated the potential of CBTp to prevent psychosis when an individual begins to experience symptoms. Van der Gaag et al demonstrated that for CBT-based interventions there is reduced transition to psychosis (relative risk $=0.52$ ) with a number needed-to-treat (NNT) of $13(95 \% \mathrm{Cl} 7$ to 71$)$ at 12 months ${ }^{14}$; Hutton and Taylor $^{15}$ found less transition at 6,12 and 18 months, with an NNT of 20. The new National Institute for Health and Care Excellence (NICE) guidelines also suggest using CBTp as the first-line treatment in at-risk groups. ${ }^{16}$

CBTp is still rapidly moving forward. We now have combination treatments to get maximal effects (eg, motivational interviewing, ${ }^{17}$ cognitive remediation ${ }^{18}$ ) and also the inclusion of new groups of individuals. For instance in a paradigm changing study for people with continuing symptoms but who refuse medication, Morrison et a ${ }^{19}$ demonstrated successful treatment by CBTp. This is the first time such an RCT has been carried out and the authors are to be commended on their rigour and particularly on reporting adverse events (2 in the CBTp group vs 6 in the control group). The study challenges the belief that CBTp is only an adjunct to medication treatment in more chronic populations - although we cannot yet conclude that CBTp should be recommended until we have more and larger trials.

\section{SO IS CBTP EFFECTIVE?}

All the meta-analyses described here were carried out rigorously. They allow us to draw a picture of a therapy that developed from its roots in medication-resistant positive symptoms to the wealth of new targets and then to the more radical-the prevention of psychosis. Only one recent meta-analysis does not show beneficial effects after accounting for methodology. This odd one out is odd for very good reasons and provides us with a backdrop to understand the treatments required in the future.

We cannot now consider CBTp as one global brand. Not all CBTps are the same. They differ in their models, the length of treatment, their targets and, probably importantly, the types of individuals who can benefit from therapy. One recommendation based on this corpus of data is that treatment allocation should take into account patient defined goals and critical targets that limit recovery in choosing the most appropriate CBTp from the current recipe book.

Acknowledgements Til Wykes would like to acknowledge the support of the NIHR BRC in mental health at the South London and Maudsley NHS Foundation Trust and the Institute of Psychiatry, KCL. She would also like to acknowledge her NIHR Senior Investigator Award.

\section{Competing interests None}

doi:10.1136/eb-2014-101887

\section{REFERENCES}

1. Garety PA, Kuipers E, Fowler D, et al. A cognitive model of the positive symptoms of psychosis. Psychol Med 2001;31:189-95.

2. Wykes T, Hayward P, Thomas $\mathrm{N}$, et al. What are the effects of group cognitive behaviour therapy for voices? A randomised control trial. Schizophr Res 2005:77:201-10.

3. Turkington D, Kingdon D, Rathod $\mathrm{S}$, et al. Outcomes of an effectiveness trial of cognitive-behavioural intervention by mental health nurses in schizophrenia. $\mathrm{Br} \mathrm{J}$ Psychiatry 2006;189:36-40.

4. Wykes T, Steel C, Everitt B, et al. Cognitive behavior therapy for schizophrenia: effect sizes, clinical models, and methodological rigor. Schizophr Bull 2008;34:523-37.

5. Steel C, Tarrier N, Stahl D, et al. Cognitive behaviour therapy for psychosis: the impact of therapist training and supervision. Psychother Psychosom 2012;81:194-5.

6. Grant PM, Huh GA, Perivoliotis D, et al. Randomized trial to evaluate the efficacy of cognitive therapy for low-functioning patients with schizophrenia. Arch Gen Psychiatry 2012;69:121-7.

7. Fowler D, Hodgekins J, Painter $M$, et al. Cognitive behaviour therapy for improving social recovery in psychosis: a report from the ISREP MRC Trial Platform study (improving social recovery in early psychosis). Psychol Med 2009;39:1627-36.

8. Birchwood M, Michail M, Meaden A, et al. Cognitive behaviour therapy to prevent harmful compliance with command hallucinations (COMMAND): a randomised controlled trial. The Lancet Psychiatry 2014;1:22-33.

9. Jauhar $\mathbf{P E}$, et al. An oversimplification of psychosis, its treatment, and its outcomes? BJP January. E-letter, 16 May 2014. http://bjp.rcpsych.org/content/204/ 1/20/reply\#bjprcpsych el 55218

10. Jauhar S, McKenna PJ, Radua J, et al. Cognitive-behavioural therapy for the symptoms of schizophrenia: systematic review and meta-analysis with examination of potential bias. Br J Psychiatry 2014;204:20-9.

11. Burns AM, Erickson DH, Brenner CA. Cognitive-behavioral therapy for medication-resistant psychosis: a meta-analytic review. Psychiatr Serv 2014. Published Online First. doi: 10.1176/appi.ps.201300213

12. van der Gaag $\mathbf{M}$, Valmaggia $L R$, Smit $F$. The effects of individually tailored formulation-based cognitive behavioural therapy in auditory hallucinations and delusions: a meta-analysis. Schizophr Res 2014;156:30-7.

13. Turner DT, van der Gaag M, Karyotaki $\mathrm{E}$, et al. Psychological interventions for psychosis: a meta-analysis of comparative outcome studies. Am J Psychiatry 2014;171:523-38.

14. van der Gaag $\mathbf{M}$, Smit F, Bechdolf $A$, et al. Preventing a first episode of psychosis: meta-analysis of randomized controlled prevention trials of 12 month and longer-term follow-ups. Schizophr Res 2013;149:56-62.

15. Hutton P, Taylor PJ. Cognitive behavioural therapy for psychosis prevention: a systematic review and meta-analysis. Psychol Med 2014;44:449-68.

16. NICE. Psychosis and schizophrenia in adults: treatment and management. NICE clinical guideline 178-Issued: February 2014. http://www.nice.org.uk/CG178

17. Barrowclough C, Haddock g, Wykes T, et al. Integrated motivational interviewing and cognitive behavioural therapy for people with psychosis and comorbid substance misuse: randomised controlled trial. BMJ 2010:341:c6325.

18. Drake RJ, Day CJ, Picucci R, et al. A naturalistic, randomized, controlled trial combining cognitive remediation with cognitive-behavioural therapy after first-episode non-affective psychosis. Psychol Med 2014;44:1889-99.

19. Morrison AP, Pyle M, Chapman N, et al. Metacognitive therapy in people with a schizophrenia spectrum diagnosis and medication resistant symptoms: a feasibility study. J Behav Ther Exp Psychiatry 2014;45:280-4. 